\title{
Chicken faecal microbiota and disturbances induced by single or repeated therapy with tetracycline and streptomycin
}

\author{
Petra Videnska, Marcela Faldynova, Helena Juricova, Vladimir Babak, Frantisek Sisak, Hana Havlickova
} and Ivan Rychlik*

\begin{abstract}
Background: In this study, we characterised the microbiota present in the faeces of 15- and 46-week-old egg laying hens before and after tetracycline or streptomycin therapy. In the first experiment, the layers were subjected to 7 days of therapy. In the second experiment, the hens were subjected to two days of therapy, which was repeated for an additional two days after 12 days of antibiotic withdrawal. This enabled us to characterise dynamics of the changes after antibiotic administration and withdrawal, and to identify genera repeatedly resistant to tetracycline and streptomycin.

Results: Real-time PCRs specific for Enterobacteriales, Lactobacillales, Clostridiales and Bifidobacteriales showed that changes in the microbiota in response to antibiotic therapy and antibiotic withdrawal were quite rapid and could be observed within 24 hours after the change in therapy status. Pyrosequencing of PCR amplified V3/N4 variable regions of $16 \mathrm{~S}$ rRNA genes showed that representatives of the orders Clostridiales, Lactobacillales, Bacteroidales, Bifidobacteriales, Enterobacteriales, Erysipelotrichales, Coriobacteriales, Desulfovibrionales, Burkholderiales, Campylobacterales and Actinomycetales were detected in the faeces of hens prior to the antibiotic therapy. Tetracycline and streptomycin therapies decreased the prevalence of Bifidobacteriales, Bacteroidales, Clostridiales, Desulfovibrionales, Burkholderiales and Campylobacterales in faecal samples in both experiments. On the other hand, Enterobacteriales and Lactobacillales always increased in prevalence in response to both therapies. Within the latter two orders, Escherichia and Enterococcus were the genera prevalence of which increased after all the antibiotic treatments.
\end{abstract}

Conclusions: The changes in microbiota composition induced by the antibiotic therapy were rapid and quite dramatic and only representatives of the genera Enterococcus and Escherichia increased in response to the therapy with both antibiotics in both experiments.

Keywords: Chicken, Microbiome, Intestinal tract, Pyrosequencing, Tetracycline, Streptomycin

\section{Background}

The gut microbiology of Gallus gallus has received considerable prior attention, however, the majority of experiments have been performed with broilers, and the gut microbiota composition in egg laying hens has been characterised much less frequently [1-4]. The reason why the majority of experiments have been performed in broilers is quite clear, as the gut microbiota, especially its altered development, significantly reduces the profitability of broiler producers [5].

\footnotetext{
*Correspondence: rychlik@vri.cz

Veterinary Research Institute, Hudcova 70, 621 00, Brno, Czech Republic
}

The whole issue of the development of gut microbiota in newly hatched chickens is further complicated by a total absence of hens as donors of healthy microbiota during the hatching of chickens in commercial production. The gut colonisation of chickens in commercial production immediately after hatching is therefore dependent on environmental sources only. The first colonisers usually belong to Enterobacteriales followed by representatives of Clostridiales and Lactobacillales [1,3,6-8]. Our unpublished results show that these initial colonisers are frequently resistant to commonly used antibiotics such as tetracycline, streptomycin or ampicillin. The initial

\section{Ciomed Central}

(c) 2013 Videnska et al.; licensee BioMed Central Ltd. This is an Open Access article distributed under the terms of the Creative Commons Attribution License (http://creativecommons.org/licenses/by/2.0), which permits unrestricted use, distribution, and reproduction in any medium, provided the original work is properly cited. 
colonisation may therefore lead to the establishment of antibiotic resistant microbiota followed by a prolonged persistence of antibiotic resistant bacteria in the intestinal tract of chickens. However, for how long and how easily such resistant clones can be positively selected for by antibiotic therapy later during chicken life, is relatively unknown. The results obtained from humans or mice indicate that the changes induced by antibiotic therapy are quite severe but relatively soon after withdrawal of the therapy, within 2 weeks, the microbiota composition returns back to the state prior to the therapy [9-12]. In this study we therefore characterised the chicken faecal microbiome and changes induced by antibiotic therapy. The obtained results allowed us to identify bacterial genera present in the chicken gut microbiome, and out of these the genera which were repeatedly resistant to both streptomycin and tetracycline therapy and could therefore serve as reservoirs and potential donors of antibiotic resistance to other bacterial species.

\section{Results}

Composition of faecal microbiota in layers subjected to single-cycle therapy determined by real-time PCR

The administration of streptomycin or tetracycline to 15-week-old layers resulted in an increased representation of Enterobacteriales and a decreased representation
Bifidobacteriales, though an increased ratio of Enterobacteriales to total bacteria after streptomycin therapy was true for one time point only. Both these antibiotics had a similar activity on Lactobacillales, which increased in representation after termination of the therapy, despite a transient decrease in Lactobacillales immediately after the tetracycline treatment. Tetracycline also decreased representation of Clostridiales whereas streptomycin therapy did not influence the representation of Clostridiales considerably (Figure 1).

\section{Composition of faecal microbiota in layers subjected to repeated-cycle therapy determined by real-time PCR}

Since the results in the previous experiment showed that significant changes in gut microbiota occur as early as two days after the initiation of antibiotic therapy, in the second experiment we subjected the hens only to a two-day therapy. In addition, we tested the consequences of repeated cycles of antibiotic therapy. Tetracycline therapy caused an increase in Enterobacteriales after the initial therapy but had no effect on Enterobacteriales immediately after the repeated therapy. Lactobacillales were resistant to both the streptomycin and tetracycline therapies, and their representation did not exhibit any clear response profile. Streptomycin and tetracycline therapies tended to decrease the representation of Bifidobacteriales and Clostridiales immediately after

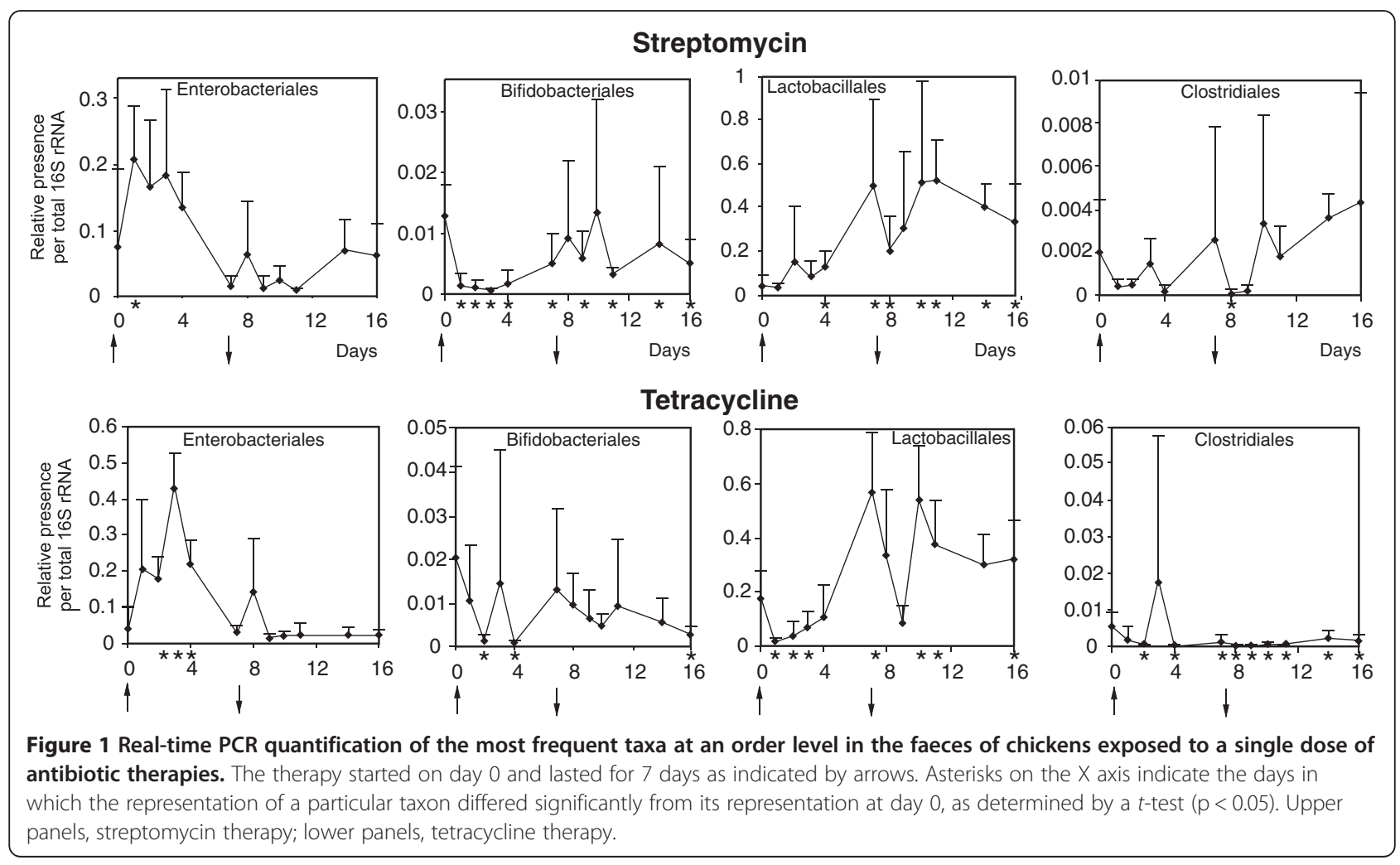


primary or repeated streptomycin and tetracycline administration, but soon after antibiotic withdrawal, an increase in the representation of these orders associated with a considerable day-to-day fluctuation was observed (Figure 2).

\section{Faecal microbiota prior to therapy determined by 454 pyrosequencing in both experiments}

To obtain a deeper insight into the changes occurring to the faecal chicken microbiota in response to the antibiotic therapy, pyrosequencing of the V3/V4 regions of the $16 \mathrm{~S}$ rRNA genes was undertaken. In between 14,446 and 76,785 independent sequences were obtained for 11 different samples. Representatives of 13 phyla were detected at least once during the 2 experiments (Table 1 and Additional file 1: Table S1), however, the vast majority of the microbiota, over 99\%, was formed by representatives of 4 phyla only; Firmicutes, Bacteroidetes, Proteobacteria and Actinobacteria. The relative representation of individual phyla in the faeces differed prior to antibiotic therapy between the 2 experiments. In the single-cycle therapy experiment, a slightly lower level of Firmicutes and higher levels of Proteobacteria and Actinobacteria were observed when compared with the repeated-cycle therapy experiment (Figure 3 ). The difference in the initial status of the chicken faecal microbiota in both the experiments was even more obvious at the order level. In the first experiment, Clostridiales dominated over the representatives of the remaining orders whereas the faecal microbiota of hens in the second experiment with repeated antibiotherapy was dominated by Lactobacillales (Figure 3).

\section{The complexity of faecal microbiota after antibiotic} therapy determined by $\mathbf{4 5 4}$ pyrosequencing

Therapy with both antibiotics in both experiments reduced the complexity of gut microbiota two days after antibiotic therapy. Chao1 index estimated the total number of OTUs prior the therapy in the layers in the first experiment to 769 and 887 which decreased to 209 and 240 after two days of streptomycin and tetracycline therapy, respectively. Prior the second experiment with the repeated antibiotic therapy, Chaol index estimated the total number of OTUs present in faeces to 1,791 which decreased to 709 and 290 after two days of streptomycin and tetracycline therapy, respectively. The interruption of antibiotic therapy for 12 days allowed for a rapid recovery of microbiota since Chaol index increased to 1,043 and 1,510 after streptomycin and tetracycline therapy, respectively. However, the repeated antibiotic administration decreased the microbiota complexity again as the Chao 1 index decreased to 422 and 357 after repeated streptomycin or tetracycline therapies, respectively (Table 1).

UniFrac $\beta$-diversity analysis followed by PCoA (principal co-ordinate analysis) indicated a clear separation between

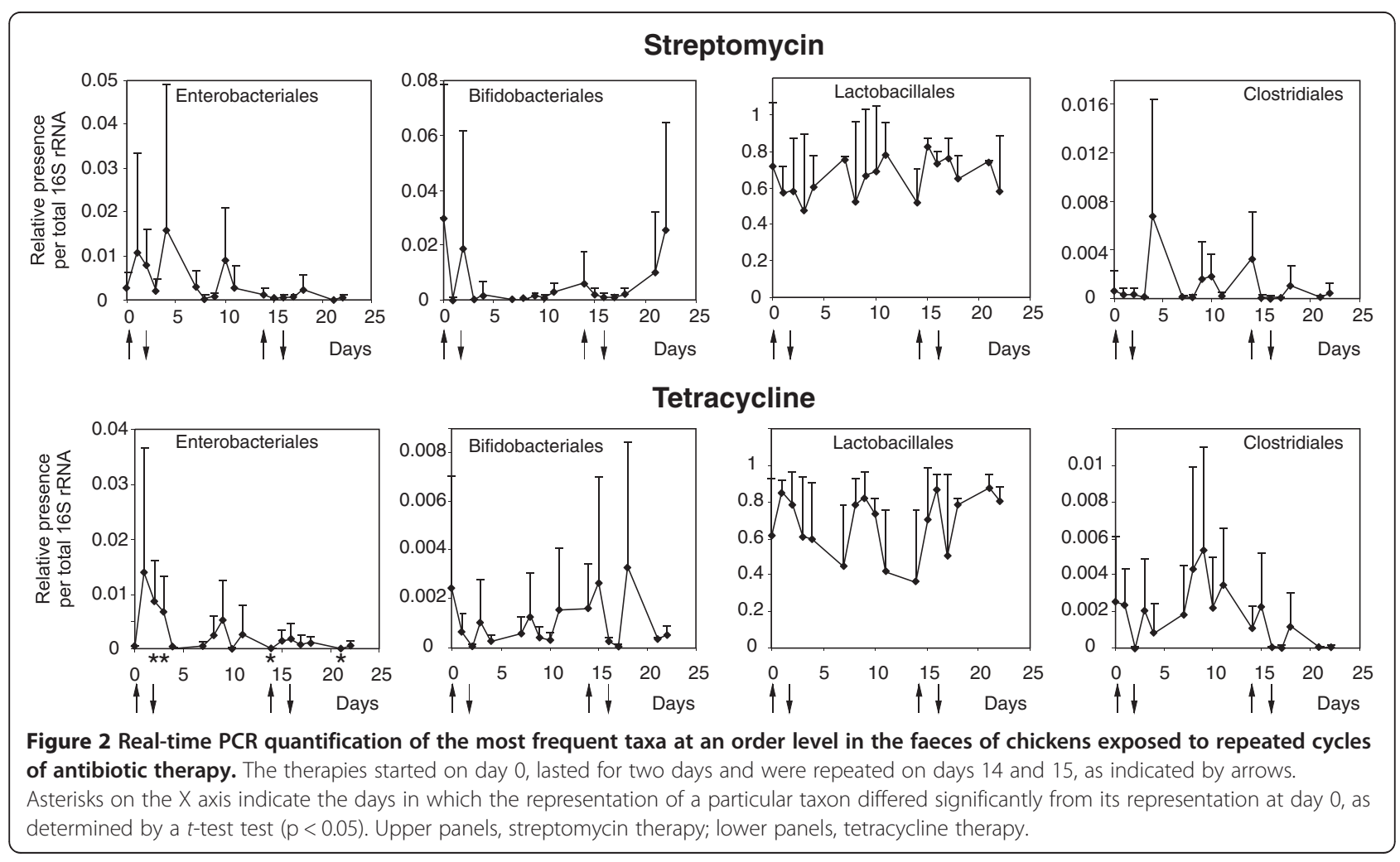


Table 1 The relative representation of individual phyla (in $\%$ of total microbiota) prior to, during and after the antibiotic therapy

\begin{tabular}{|c|c|c|c|c|c|c|c|c|c|c|c|}
\hline \multirow[b]{2}{*}{ Day } & \multicolumn{4}{|c|}{ Experiment 1} & \multicolumn{7}{|c|}{ Experiment 2} \\
\hline & 0 & 0 & 2 & 2 & 0 & 2 & 2 & 14 & 14 & 16 & 16 \\
\hline phyllum & Str & Tet & Str & Tet & $\mathrm{Str} / \mathrm{Tet}$ & Str & Tet & Str & Tet & Str & Tet \\
\hline Bacteria. other & 0.02 & 0.02 & 0.02 & 0.02 & 0.02 & 0.01 & ND & 0.09 & 0.46 & ND & ND \\
\hline Actinobacteria & 7.21 & 4.09 & 0.38 & 0.09 & 1.67 & 8.7 & 0.01 & 0.35 & 1.76 & 0.24 & 0.11 \\
\hline Bacteroidetes & 13.04 & 3.56 & 0.07 & 0.02 & 6.61 & 0.57 & ND & 2.48 & 11.3 & 0.04 & ND \\
\hline Cyanobacteria & 0.01 & ND & 0.02 & 0.01 & ND & ND & ND & ND & ND & ND & ND \\
\hline Deferribacteres & ND & ND & ND & ND & 0.02 & ND & ND & ND & 0.23 & ND & ND \\
\hline Firmicutes & 61.83 & 86.60 & 45.07 & 27.81 & 89.88 & 90.3 & 99.88 & 89.84 & 82.94 & 99.22 & 99.8 \\
\hline Fusobacteria & ND & ND & ND & ND & 0.77 & 0.12 & ND & 6.81 & 0.24 & 0.4 & ND \\
\hline Chloroflexi & ND & ND & 0.003 & ND & ND & ND & ND & ND & ND & ND & ND \\
\hline Lentisphaerae & ND & ND & ND & ND & 0.003 & ND & ND & ND & ND & ND & ND \\
\hline Proteobacteria & 17.80 & 5.33 & 54.41 & 72.04 & 0.9 & 0.16 & 0.08 & 0.28 & 2.2 & 0.03 & 0.04 \\
\hline Spirochaetes & ND & ND & ND & ND & 0.001 & ND & ND & ND & ND & ND & ND \\
\hline Synergistetes & ND & ND & ND & ND & 0.1 & 0.07 & ND & 0.11 & 0.82 & ND & ND \\
\hline Tenericutes & ND & ND & ND & ND & 0.001 & ND & ND & ND & ND & ND & ND \\
\hline TM7 & 0.09 & 0.39 & 0.01 & ND & 0.01 & 0.02 & ND & 0.01 & ND & 0.01 & ND \\
\hline OTU* & 590 & 709 & 172 & 273 & 2222 & 433 & 182 & 896 & 1028 & 236 & 228 \\
\hline Chaol & 1106 & 932 & 488 & 263 & 4592 & 709 & 263 & 1675 & 1860 & 490 & 364 \\
\hline OTU 14,446 & 478 & 528 & 131 & 182 & 869 & 433 & 164 & 541 & 799 & 211 & 165 \\
\hline Chaol 14,446 & 769 & 887 & 209 & 240 & 1791 & 709 & 290 & 1043 & 1510 & 422 & 357 \\
\hline Evenness & 0.615 & 0.57 & 0.3 & 0.24 & 0.37 & 0.47 & 0.2 & 0.39 & 0.45 & 0.22 & 0.28 \\
\hline Shannon index & 4.03 & 3.68 & 1.71 & 1.26 & 2.87 & 2.85 & 1.03 & 2.65 & 3.12 & 1.25 & 1.53 \\
\hline Total n. of reads & 22,885 & 29,269 & 32,010 & 25,031 & 76,785 & 14,446 & 19,834 & 37,707 & 22,615 & 17,808 & 27,703 \\
\hline
\end{tabular}

ND - not detected

* OTU - number of OTUs using all reads available for each sample, Chao1 index estimates OTUs richness; OTU 14,446 and Chao1 14,446 - as above but normalised to randomly selected 14,446 reads for each sample, i.e. to the number of reads available for the sample with the lowest coverage; "Evenness" characterises how close in numbers each OTUs were present in each microbial population; Shannon index combines both species richness and evenness of their distribution within given microbial population.
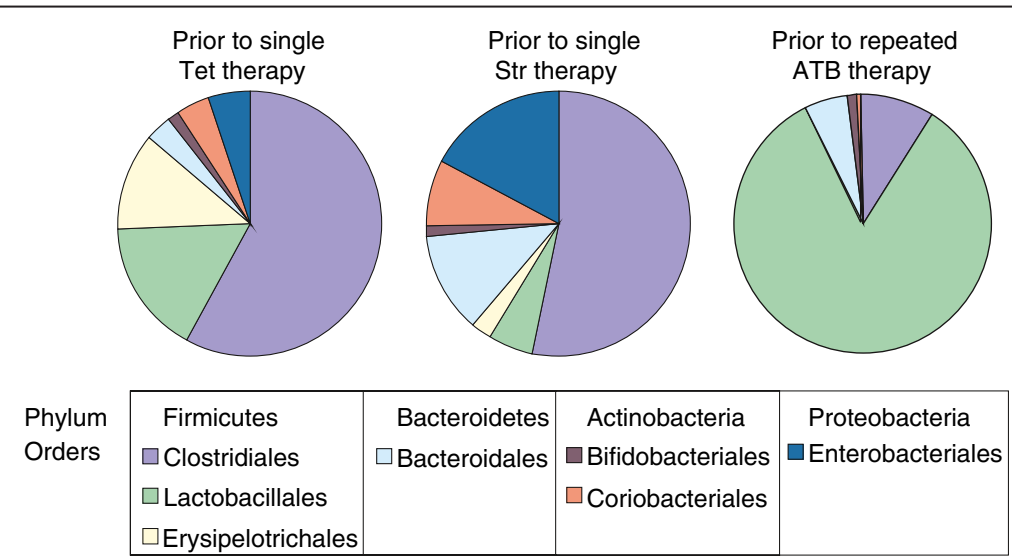

Figure 3 Composition of the main orders present in chicken faecal microbiota prior to antibiotic therapy. The sum of the appropriate orders as indicated in the figure legend provides information on the microbiota distribution at the phylum level. As in the single dose therapy experiment, the chickens were left to accommodate in two different groups, two pie charts are shown for this experiment. 
the antibiotic treated and non-treated groups using both un-weighted and weighted analysis (Figure 4). The PC1 (principal coordinate) in the un-weighted analysis, which ignores the relative representation of individual microbiota members, showed that a single factor explained $27 \%$ of all the variability among compared groups. PC1 in weighted analysis, which includes the relative representation of individual OTU into calculation, explained 52\% the variability among compared groups. Displaying the microbiota representatives in biplot $\mathrm{PCOA}$ indicated slightly lower effect of primary streptomycin treatment in the second experiment. This analysis also clustered the representatives of phyla Bacteroidetes and Actinobacteria with the nontreated layers and Proteobacteria with the antibiotic-treated layers (Figure 4).

Analysis at the lower taxonomical levels showed that the therapy with both antibiotics in both the experiments always reduced the prevalence of Bifidobacteriales, Bacteroidales, Clostridiales, Desulfovibrionales, Burkholderiales and Campylobacterales. On the other hand, the orders Enterobacteriales and Lactobacillales increased in relative representation after the administration of both tetracycline and streptomycin in both experiments (Additional file 1: Table S1). When we analysed the composition of the orders in which the increase in prevalence was recorded in both experiments at the genus level, Enterobacteriales was comprised of representatives of the genera Pantoea, Proteus, Citrobacter, Enterobacter and Escherichia. However, since around 98\% of all Enterobacteriales were formed by Escherichia, the increase observed for the whole order after both streptomycin and tetracycline administration was caused by the representatives of genus Escherichia. The order Lactobacillales comprised of 10 different genera, out of which the genera Lactobacillus, Enterococcus, Paralactobacillus and Streptococcus formed more than 99\% of all Lactobacillales. However, only representatives of the genus
Enterococcus increased in both experiments and after the therapies with both antibiotics.

\section{Microbiota composition along the chicken digestive tract}

In the last experiment, we searched for the possible sources of microbiota which increased in representation during or after therapy. Pyrosequencing of $16 \mathrm{~S}$ rRNA amplification products from crop, gizzard, stomach, duodenum, ileum, caecum and colon DNA resulted in between 2,332 and 25,997 independent sequences. The crop, gizzard, stomach and small intestine were mutually quite similar in composition to each other but different from the caecum and colon. The crop was dominated by Lactobacillus followed by Gallibacterium (family Pasteurellaceae). The less abundant genera in the crop included Veillonella and Enterococcus. Feacalibacterium and Bacteroides were detected in the stomach although these genera were otherwise characteristic for the caecum and colon. Both parts of the small intestine were dominated by Lactobacillus species. The diversity of microbiota considerably increased in the caecum and colon (Chao1 OTU estimates predicted 342 different OTUs in the crop, 1,028 in the gizzard, 733 in the stomach, 1,223 in the duodenum, 169 in the ileum, 1,821 in the caecum and 4,647 in the colon) with different strict anaerobes forming the majority of microbiota members (Figure 5 and Additional file 2: Table S2).

\section{Discussion}

In this study we characterised chicken faecal microbiota and changes induced by antibiotic therapy by real time PCR and pyrosequencing of $16 \mathrm{~S}$ rRNA amplification products. The results from the real time PCR must be considered with increased care as it was rather difficult to design primer pairs specific for orders as diverse as Clostridiales. This can be clearly seen if data from Figure 1 and 2 are compared with Figure 3. If $Y$ axis scaling in Figures 1 and 2 is multiplied by a factor of 100, the same
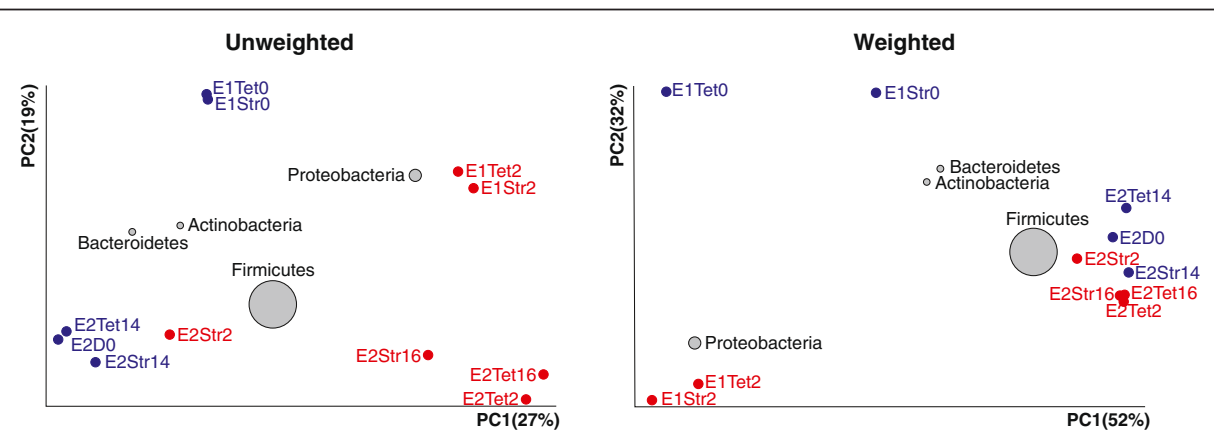

Figure 4 Unweighted and weighted UniFrac PCoA of faecal microbiota from hens with and without antibiotic treatment. Diameter of the circle for 4 major phyla present in the faeces of chickens corresponds to their average relative representation in all the samples. Sample designation - E1 or E2, experiment with single or repeated therapy; Str" $n$ " or Tet" $n$ ", streptomycin or tetracycline therapy where " $n$ " indicates number of days from the beginning of the experiment. Blue designations, microbiomes of chicken prior therapy, red designations, microbiomes of chickens two days after therapy. 


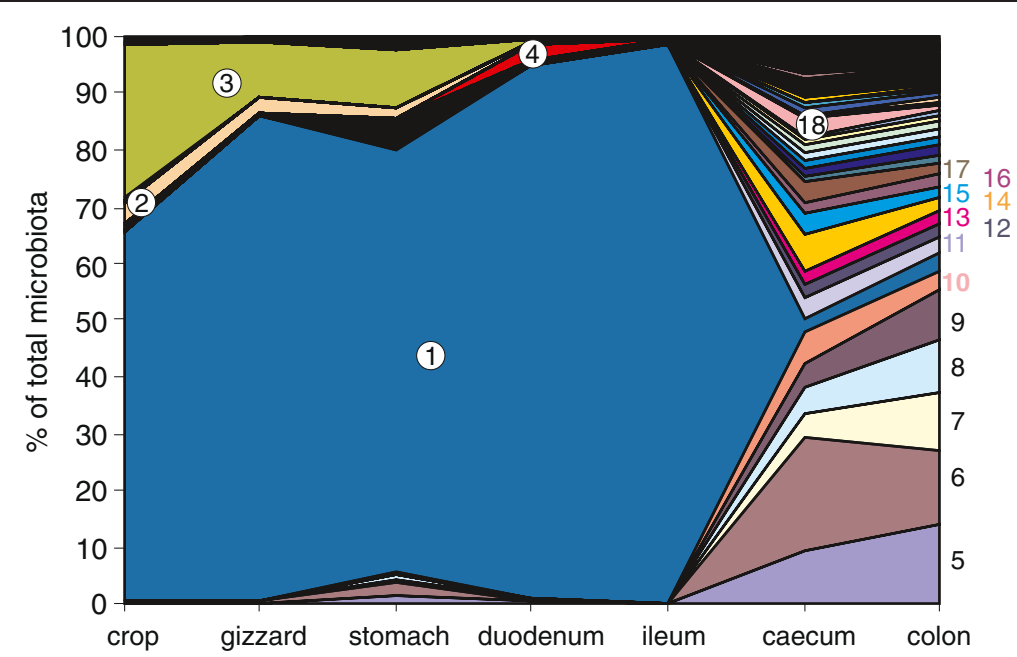

Figure 5 Species distribution along the digestive tract of an adult hen. Only the most frequent genera are identified. For complete data, see Additional file 2: Table S2. 1 - Lactobacillus, 2 - Veillonella, 3 - Gallibacterium, 4 - Campylobacter, 5 - unclassified Lachnospiraceae, 6 - Bacteriodes, 7 - Faecalibacterium, 8 - Megamonas, 9 - Olsenella, 10 - Phascolarctobacterium, 11 - Prevotella, 12 - Blautia, 13 - Pseudoflavonifractor, 14 - Barnesiella, 15 - Desulfovibrio, 16 - Clostridium XIVa, 17 - unclassified Porphyromonadaceae, 18 - Alistipes.

percentage out of total microbiota as in Figure 3 can be deduced. Such comparison shows that real time PCR detection of Enterobacteriales and Lactobacillales provided similar data as pyrosequencing but Bifidobacteriales and Clostridiales were highly underestimated by the real time PCR. Being aware of this, we used the real time PCR mainly to characterise time-dependent changes in microbiota composition, which should be less affected by the relaxed specificity of the 2 real time PCRs, and also to obtain some information on hen-to-hen variation.

The composition of faecal microbiota prior to the antibiotic therapy was similar to that reported in previous studies in chickens i.e. Firmicutes dominating, followed by Bacteroidetes and Proteobacteria [1,4,7]. In the 15-week layers, there was a higher proportion of Proteobacteria at the expense of Firmicutes when compared with the 46-week old hens, which may correlate with known gradual colonisation patterns in young animals $[8,13]$ but may also be a consequence of the 3-week adaptation to a new, clean experimental animal house prior to the first experiment.

The changes in microbiota composition induced by the antibiotic therapy were rapid and quite dramatic as in between 55 (streptomycin therapy in the first experiment) to $94 \%$ (first tetracycline administration during the second experiment) of OTU disappeared or decreased below the detection limit within 48 after antibiotic administration. Only representatives of the genera Enterococcus and Escherichia increased in response to the therapy with both antibiotics in both experiments, despite the fact that layers of different age and with different microbiota composition were used in the two experiments. However, one has to be reminded that the increases reported in this study may not necessarily correlate with the increase in total bacterial counts of the appropriate taxon. If streptomycin or tetracycline inactivated certain groups of bacteria but left the others unaffected, the latter will increase in proportion but not in actual numbers. Since the chickens or hens were kept under the same conditions, all the birds were provided the same feed and for the duration of the experiment, they were kept in animal house with air conditioning and strict hygienic regime minimising the external sources of microbiota to technical minimum, we did not include a control non-treated group. We believe that such extensive changes in gut microbiota upon antibiotic administration were direct consequences of the therapy and not of random fluctuation in gut microbiota composition.

Interestingly, the origin of the representatives of Enterococcus and Escherichia could be quite different. Although we performed the microbiota characterisation along the digestive tract only in a single set of samples, representatives of Enteroccocus were present only in the proximal parts of the digestive tract (crop, gizzard, stomach) while representatives of Escherichia were found in caecum or colon. On the other hand, sources of Lactobacillus which increased in faeces in the single cycle therapy experiment could originate from the crop till the jejunum. Not surprisingly we observed the increase in Lactobacillus prevalence in faeces only in the single-dose therapy experiment where a low representation of Lactobacillus was observed prior to the therapy, leaving a space for Lactobacillus increase after the therapy withdrawal. This could not happen in the experiment with repeated therapy as in this case, the Lactobacillus representation was quite high prior to antibiotic therapy not allowing for an additional increase. The 
restoration of microbiota complexity after therapy withdrawal was nearly as rapid as the changes immediately after the therapies. Twelve days after the withdrawal, the estimated number of OTUs increased and PCoA analysis clustered microbiomes of such layers with that of the nontreated layers. One of the potential reservoirs for such a rapid microbiota restoration could be found in the stomach, since in this organ we found certain microbiota members which were otherwise common to caecum, colon or faeces.

\section{Conclusions}

Although the experiments described in this study have been performed on a limited number of hens and in pooled samples, we observed that the changes in microbiota composition induced by the antibiotic therapy were rapid and quite dramatic and only representatives of the genera Enterococcus and Escherichia increased in response to the therapy with both antibiotics in both experiments. Interestingly, the restoration of microbiota complexity after therapy withdrawal was nearly as rapid as the changes immediately after the therapies. The stomach can be understood as one of the possible reservoirs for such a rapid microbiota restoration since certain microbiota members, which were otherwise common to caecum, colon or faeces, were found also in this organ.

\section{Methods}

\section{Experimental animals}

Female Lohmann Brown layers, obtained from a commercial producer with no history of antibiotic use, were used in this study. The handling of animals in the study was performed in accordance with current Czech legislation. The specific experiments were approved by the Ethics Committee of the Veterinary Research Institute followed by the Committee for Animal Welfare of the Ministry of Agriculture of the Czech Republic.

\section{Single-cycle therapy}

Twelve-week-old layers were transferred from a farm and allowed to adapt in the experimental animal house for 3 weeks prior to the antibiotic therapy. During the adaptation period, the layers were divided into two groups kept at separate rooms, each group consisting of 5 layers. Daily water consumption of the groups of 5 birds was determined and this information was used to provide layers with the antibiotics in the drinking water at such a concentration that the daily uptake was equivalent to $60 \mathrm{mg}$ of tetracycline or $15 \mathrm{mg}$ of streptomycin per $\mathrm{kg}$ of body weight, respectively. The drinking water with antibiotics was administered successively for 7 days and faecal samples were individually collected from each layer. The first sampling was performed when the layers were 15 -week old, just prior to the antibiotic administration (day 0 ), followed by sampling on days $1,2,3,4,7,8,9,10$, 11,14 and 16 , when the experiment was terminated.

\section{Repeated-cycle therapy}

The aim of this experiment was to characterise the changes in the faecal microbiota of hens subjected to repeated cycles of antibiotic therapy. Two groups of five, 46-week-old hens were brought to the institute, housed in separate rooms, subjected to antibiotic therapy without any adaptation with the same antibiotics and at the same dosage as in single-cycle experiment. Furthermore, since in the first experiment we found that the greatest changes in gut microbiota were observed as early as two days after the antibiotic therapy, we treated the hens with the antibiotics for two days only, let them recover for 12 days without antibiotic administration, and on day 14 , the therapy was repeated for additional two days. Faecal samples were individually collected on days 0,1 , $2,3,4,7,8,9,10,11,14,15,16,17,18,21$ and 22, when the experiment was terminated.

\section{Characterisation of microbiota along the digestive tract}

Three adult 46-week-old hens were sacrificed and the contents of the crop, gizzard, stomach, duodenum, ileum, caecum and colon were taken for DNA purification. The composition of microbiota was determined in all the samples from all 3 birds by real-time PCR (see below), and the samples from the hen exhibiting the median values in most of the real time PCRs were subjected to pyrosequencing of the $16 \mathrm{~S}$ rRNA amplification products (see below).

\section{DNA purification and taxon-specific real-time PCR}

DNA was extracted from the faeces by QIAamp DNA Stool Mini Kit according to the manufacturer's instructions (Qiagen) and stored at $-20^{\circ} \mathrm{C}$ until use. Taxon-specific primers were designed from the variable regions of $16 \mathrm{~S}$ rRNA genes with PRIMROSE software [14] and the specificity of the primers was tested by RDP ProbeMatch program. Two primer pairs specific for the conservative regions of $16 \mathrm{~S}$ rRNA genes (domain Bacteria universal primer pairs) served to determine the total bacterial DNA present in the samples (Table 2). Real-time PCR was carried out using QuantiTect SYBR Green PCR Kit (Qiagen) in a LightCycler LC480 thermocycler (Roche). The PCR was initiated with a hot start for $15 \mathrm{~min}$ at $95^{\circ} \mathrm{C}$ followed by 45 cycles of $20 \mathrm{sec}$ at $95^{\circ} \mathrm{C}, 30 \mathrm{sec}$ at $60^{\circ} \mathrm{C}$ and $30 \mathrm{sec}$ at $72^{\circ} \mathrm{C}$. Melting temperatures were determined after PCR to verify the correctness of each PCR product. After PCR, the $\mathrm{Ct}$ values of the genes of interest were subtracted from an average $\mathrm{Ct}$ value of amplifications performed with universal primer pairs for the domain Bacteria $(\Delta \mathrm{Ct})$. The relative amount of each taxon in the total bacterial population was finally calculated as $2^{-\Delta \mathrm{Ct}}[15,16]$. 
Table 2 List of primers used in this study

\begin{tabular}{llll}
\hline Primer & Target & Sequence 5'- 3' & Reference \\
\hline 16S_Bifido-F & Bifidobacteriales & GGTGTGAAAGTCCATCG & this study \\
16S_Bifido-R & Bifidobacteriales & ACCGGGAATCCAGTCT & this study \\
16S_Clost-F & Clostridiales & GCGTATCCGGATTTAC & this study \\
16S_Clost-R & Clostridiales & ACACCTAGTATTCATCG & this study \\
16S_Entero-F & Enterobacteriales & STGAGACAGGTGCTGCA & this study \\
16S_Entero-R & Enterobacteriales & AAAGGATAAGGGTTGCG & this study \\
16S_Lacto-F & Lactobacillales & CTTGAGTGCAGAAGAGG & this study \\
16S_Lacto-R & Lactobacillales & CACTGGTGTCTTCCAT & this study \\
16S_univ-1F & all bacteria & GTGSTGCAYGGTGTCGTCA & {$[17]$} \\
16S_univ-1R & all bacteria & ACGTCRTCCMCACCTTCCTC & {$[17]$} \\
16S_univ-2 F & all bacteria & GAGGAAGGIGIGGAIGACGT & {$[13]$} \\
16S_univ-2R & all bacteria & AGICCCGIGAACGTATTCAC & {$[13]$} \\
\hline
\end{tabular}

\section{Pyrosequencing}

Equal amounts of faecal DNA originating from the layers of the same treatment group and time were pooled prior to PCR and used as a template in PCR with forward primer $5^{\prime}$-CGTATCGCCTCCCTCGCGCCATCAG-MIDGGAGGCAGCAGTRRGGAAT- $3^{\prime}$ and reverse primer $5^{\prime}$ CTATGCGCCTTGCCAGCCCGCTCAG-MID-CTACCR GGGTATCTAATCC-3'. The underlined sequences were required at different steps of amplicon pyrosequencing. The sequences in italics are specific to the conserved sequences of bacterial 16S rRNA genes allowing amplification of the V3/V4 hypervariable region [18]. MID represents different $10 \mathrm{bp}$ sequences which enable simultaneous sequencing and re-identification of DNA originating from different samples. After PCR, the amplification products were loaded onto a $1.2 \%$ agarose gel, separated by gel electrophoresis and gel-purified using a QIAEX II Gel Extraction Kit (Qiagen). Pyrosequencing was performed using a GS Junior 454 sequencer and GS Junior Titanium sequencing chemistry exactly according to the manufacturer's instructions (Roche). In one sequencing run, the amplification products from 4 samples were mixed and analysed.

\section{Sequence analysis}

The FASTA and qual files generated as an output of pyrosequencing were uploaded into Qiime software [19]. Quality trimming criteria included no mismatch in the bar code sequences and maximally 1 mismatch in the primer sequences. In the next step, chimeric sequences were predicted and excluded from the analysis. The obtained sequences were then classified with RDP Seqmatch with an OTU (operational taxonomic units) discrimination level set up to $97 \%$. Diversity analyses
(Chao1 richness, Evenness estimation and Shannon index) on OTU clusters were performed using all sequences available for each sample. Finally, UniFrac analysis [20] followed by principal coordinate analysis and Biplot data visualisation was used to characterise diversity in the microbial populations tested.

\section{Statistics}

Data from real-time PCR are presented as average \pm SD. The comparison of taxon representation at a particular day to the representation on day 0, i.e. prior to antibiotic therapy, was evaluated by a $t$-test using SPSS v.14 statistical software.

\section{Additional files}

Additional file 1: Table S1. List of all reads and OTUs identified after antibiotic treatment in both experiments of this study.

Additional file 2: Table S2. List of all reads and OTUs identified along digestive tract of adult hen.

\section{Competing interests}

The authors state that they do not have and financial or personal conflicts that could inappropriately bias their work.

\section{Authors' contributions}

PV performed the pyrosequencing, analysed the data and helped to draft the manuscript. MF and HJ purified the DNA, designed real time PCR primers and performed the real time PCR. FS and $\mathrm{HH}$ were responsible for the animal experiments and sample collection. VB performed the statistical analysis. IR participated in the design of the study, data analysis and helped to draft the manuscript. All authors read and approved the final manuscript.

\section{Acknowledgement}

This work has been supported by the projects MZE0002716202 of the Czech Ministry of Agriculture, AdmireVet project CZ.1.05/2.1.00/01.0006 - ED0006/ 01/01 from the Czech Ministry of Education and EMIDA HealthyGut project. Authors would like to thank Peter Eggenhuizen for his English language corrections and Prof. Paul O'Toole for his introduction to the Qiime software.

Received: 27 September 2012 Accepted: 9 February 2013

Published: 13 February 2013

\section{References}

1. Zhu XY, Zhong T, Pandya Y, Joerger RD: $16 \mathrm{~S}$ rRNA-based analysis of microbiota from the cecum of broiler chickens. Appl Environ Microbiol 2002, 68:124-137.

2. Knarreborg A, Simon MA, Engberg RM, Jensen BB, Tannock GW: Effects of dietary fat source and subtherapeutic levels of antibiotic on the bacterial community in the ileum of broiler chickens at various ages. Appl Environ Microbiol 2002, 68:5918-5924.

3. Lu J, Idris U, Harmon B, Hofacre C, Maurer JJ, Lee MD: Diversity and succession of the intestinal bacterial community of the maturing broiler chicken. Appl Environ Microbiol 2003, 69:6816-6824.

4. Nordentoft S, Molbak L, Bjerrum L, De Vylder J, Van Immerseel F, Pedersen $K$ : The influence of the cage system and colonisation of Salmonella Enteritidis on the microbial gut flora of laying hens studied by T-RFLP and 454 pyrosequencing. BMC Microbiol 2011, 11:187.

5. Bokkers EA, de Boer IJ: Economic, ecological, and social performance of conventional and organic broiler production in the Netherlands. Br Poult Sci 2009, 50:546-557.

6. Amit-Romach E, Sklan D, Uni Z: Microflora ecology of the chicken intestine using 16S ribosomal DNA primers. Poult Sci 2004, 83:1093-1098.

7. Qu A, Brulc JM, Wilson MK, Law BF, Theoret JR, Joens LA, Konkel ME, Angly $F$, Dinsdale EA, Edwards RA, et al: Comparative metagenomics reveals host 
specific metavirulomes and horizontal gene transfer elements in the chicken cecum microbiome. PLoS One 2008, 3:e2945.

8. Crhanova M, Hradecka H, Faldynova M, Matulova M, Havlickova H, Sisak F, Rychlik I: Immune response of chicken gut to natural colonization by gut microflora and to Salmonella enterica serovar Enteritidis infection. Infect Immun 2011, 79:2755-2763.

9. Dethlefsen L, Huse S, Sogin ML, Relman DA: The pervasive effects of an antibiotic on the human gut microbiota, as revealed by deep $16 \mathrm{~S}$ rRNA sequencing. PLOS Biol 2008, 6:e280.

10. Antonopoulos DA, Huse SM, Morrison HG, Schmidt TM, Sogin ML, Young VB: Reproducible community dynamics of the gastrointestinal microbiota following antibiotic perturbation. Infect Immun 2009, 77:2367-2375.

11. Jernberg C, Lofmark S, Edlund C, Jansson JK: Long-term impacts of antibiotic exposure on the human intestinal microbiota. Microbiology 2010, 156:3216-3223.

12. Robinson CJ, Young VB: Antibiotic administration alters the community structure of the gastrointestinal micobiota. Gut Microbes 2010, 1:279-284

13. Tseng CP, Cheng JC, Tseng CC, Wang C, Chen YL, Chiu DT, Liao HC, Chang SS: Broad-range ribosomal RNA real-time PCR after removal of DNA from reagents: melting profiles for clinically important bacteria. Clin Chem 2003, 49:306-309.

14. Ashelford KE, Weightman AJ, Fry JC: PRIMROSE: a computer program for generating and estimating the phylogenetic range of $16 \mathrm{~S}$ rRNA oligonucleotide probes and primers in conjunction with the RDP-II database. Nucleic Acids Res 2002, 30:3481-9.

15. Juricova H, Videnska P, Lukac M, Faldynova M, Babak V, Havlickova H, Sisak F, Rychlik I: Influence of Salmonella enterica serovar Enteritidis infection on the development of cecum microbiota in newly hatched chicks. App/ Environ Microbiol 2013, 79:745-7.

16. Matulova M, Rajova J, Vlasatikova L, Volf J, Stepanova H, Havlickova H, Sisak F, Rychlik I: Characterization of chicken spleen transcriptome after infection with Salmonella enterica serovar Enteritidis. PLoS One 2012, 7:e48101.

17. Maeda H, Fujimoto C, Haruki Y, Maeda T, Kokeguchi S, Petelin M, Arai H, Tanimoto I, Nishimura F, Takashiba S: Quantitative real-time PCR using TaqMan and SYBR Green for Actinobacillus actinomycetemcomitans, Porphyromonas gingivalis, Prevotella intermedia, tetQ gene and total bacteria. FEMS Immunol Med Microbiol 2003, 39:81-86.

18. Nossa CW, Oberdorf WE, Yang L, Aas JA, Paster BJ, Desantis TZ, Brodie EL, Malamud D, Poles MA, Pei Z: Design of 16S rRNA gene primers for 454 pyrosequencing of the human foregut microbiome. World I Gastroenterol 2010, 16:4135-4144.

19. Caporaso JG, Kuczynski J, Stombaugh J, Bittinger K, Bushman FD, Costello EK, Fierer N, Pena AG, Goodrich JK, Gordon Jl, et al: QIIME allows analysis of high-throughput community sequencing data. Nat Methods 2010 7:335-336.

20. Lozupone C, Knight R: UniFrac: a new phylogenetic method for comparing microbial communities. Appl Environ Microbiol 2005, 71:8228-8235.

doi:10.1186/1746-6148-9-30

Cite this article as: Videnska et al:: Chicken faecal microbiota and disturbances induced by single or repeated therapy with tetracycline and streptomycin. BMC Veterinary Research 2013 9:30.

\section{Submit your next manuscript to BioMed Central and take full advantage of:}

- Convenient online submission

- Thorough peer review

- No space constraints or color figure charges

- Immediate publication on acceptance

- Inclusion in PubMed, CAS, Scopus and Google Scholar

- Research which is freely available for redistribution 